\title{
Lactobacillus rhamnosus attenuates intestinal inflammation induced by Fusobacterium nucleatum infection by restoring the autophagic flux
}

\author{
CAIHAN DUAN $^{1}$, XUELIAN TANG $^{1}$, WEIJUN WANG ${ }^{1}$, WEI QIAN $^{1}$, XIAOCHAO FU $^{2}$, \\ XIAOHUA DENG ${ }^{2}$, SHUNCHANG ZHOU ${ }^{3}$, CHAOQUN HAN ${ }^{1}$ and XIAOHUA HOU ${ }^{1}$
}

\begin{abstract}
${ }^{1}$ Division of Gastroenterology, Union Hospital, Tongji Medical College, Huazhong University of Science and Technology, Wuhan, Hubei 430022; ${ }^{2}$ Hubei Center of Industrial Culture Collection and Research, Wuhan, Hubei 430020;

${ }^{3}$ Division of Experimental Animals, Tongji Medical College, Huazhong University of Science and Technology, Wuhan, Hubei 430022, P.R. China
\end{abstract}

Received April 13, 2020; Accepted September 22, 2020

DOI: $10.3892 /$ ijmm.2020.4780

\begin{abstract}
Autophagy plays a dual role in the responses to the gut microflora. The present study aimed to examine the effects of Lactobacillus rhamnosus (L. rhamnosus) on Fusobacterium nucleatum ( $F$. nucleatum)-induced intestinal dysfunction and to elucidate the underlying mechanisms, with particular focus on autophagy.Inflammatory models were established by treatment with $L$. rhamnosus following $F$. nucleatum intervention using cells or a mouse model of dextran sulfate sodium (DSS)-induced acute colitis. Autophagosomes were visualized by confocal microscopy following transfection with a tandem GFP-mCherry-LC3 construct and also by transmission electron microscopy. Autophagy-associated protein levels were examined by western blot analysis and immunohistochemistry. It was observed that $F$. nucleatum induced the production of pro-inflammatory cytokines in Caco- 2 cells and aggravated DSS-induced acute colitis. The autophagic flux was impaired following infection with $F$. nucleatum. L. rhamnosus treatment attenuated the inflammation induced by $F$. nucleatum infection and effectively recovered the impaired autophagic flux. In addition, the production of pro-inflammatory cytokines induced by $F$. nucleatum was enhanced with autophagy inhibitors or the RNA interference of autophagy-related gene 16 like 1 (Atg16L1) in Caco- 2 cells. Notably, this inhibition of autophagy weakened the effects of L. rhamnosus. Finally, the PI3K/AKT/mTOR pathway
\end{abstract}

Correspondence to: Professor Xiaohua Hou or Dr Chaoqun Han, Division of Gastroenterology, Union Hospital, Tongji Medical College, Huazhong University of Science and Technology, 1277 Jiefang Avenue, Wuhan, Hubei 430022, P.R. China

E-mail: houxh@hust.edu.cn

E-mail: hcq1987912@hotmail.com

Key words: Fusobacterium nucleatum, Lactobacillus rhamnosus, autophagy, intestinal inflammation was found to be involved in this process. On the whole, the present study demonstrates that the mediation of autophagy by L. rhamnosus may be involved in the protective effects against F. nucleatum-related intestinal inflammation. Thus, L. rhamnosus treatment may prove to be a novel therapeutic strategy for F. nucleatum-realated gut disorders.

\section{Introduction}

Inflammatory bowel diseases (IBDs) are remittent and progressive inflammatory conditions that may affect the entire gastrointestinal tract (1). Multiple factors, such as genes, diet, environment and the microbiota contribute to complex etiologies (2). Among these factors, research into the microbiome is a rapidly advancing field in IBD. Accumulating evidence has indicated that the gut microbiota play a key role in the process of IBD. The microbiota in the human intestine may be closely related to the development of IBD via their influence on the physiological functions of the colorectum (3-5). However, the inflammatory pathogens which cause IBD remain incompletely explored.

It was previously demonstrated that the novel intestinal bacterium, Fusobacterium nucleatum (F. nucleatum), was associated with an enhanced gut inflammation in patients with IBD. The proportion of patients with IBD identified with $F$. nucleatum infection in the colonic tissues was higher than the controls. Moreover, F. nucleatum strains from the inflamed tissues of patients with IBD were more invasive than strains that were isolated from healthy tissue (6). However, the underlying mechanisms associated with the inflammatory pathogenesis of this bacterium remain largely unknown.

Autophagy is a 'self-eating' process that results in the breakdown of intracellular proteins or organelles for recycling within the lysosome to maintain cellular homeostasis (7). Excessive or impaired autophagy can result in pathological processes (8-11). More importantly, accumulating evidence has indicated an association between autophagy and IBD. Autophagy plays multiple roles in the pathogenesis of IBD by altering processes that include anti-inflammatory 
cytokine production, antigen presentation and the endoplasmic reticulum stress response (12). Moreover, autophagy plays an important role in the epithelial cell-autonomous mechanisms of the antibacterial defense that protects against the dissemination of intestinal bacteria (13). However, the role of autophagy in $F$. nucleatum-induced inflammation in intestinal epithelial cells has not yet been investigated, at least to the best of our knowledge.

It is widely accepted that probiotics exert beneficial effect against colitis (14-16). Probiotic supplementation appears to be potentially well tolerated, effective, and safe in patients with IBD (17). As previously demonstrated, the probiotic Lactobacillus rhamnosus (L. rhamnosus) improved the clinical symptoms of patients with mild to moderately active IBD (18). However, information on the role of L. rhamnosus and autophagy in the induction and progression during $F$. nucleatum-induced inflammation is limited. Thus, the aim of the present study was to examine the effects of L. rhamnosus on F.nucleatum-induced intestinal inflammation, and to elucidate the underlying mechanisms, with particular focus on autophagy. The present study wished to provide novel insight into $F$. nucleatum-related gut disorders.

\section{Materials and methods}

Reagents. Primary antibodies against microtubule-associated light chain 3B (LC3B), p-AKT and p-mammalian target of rapamycin (mTOR) were purchased from Cell Signaling Technology, Inc. (cat. nos. 3868, 4060 and 5536); antibodies against GAPDH, SQSTM1/p62, Atg16L1, p-p85, p85, AKT and mTOR were obtained from ABclonal (cat. nos. AC001, A7758, A1871, AP0854, A11526, A11016 and A2445); HRP-conjugated goat anti rabbit antibody was from Antgene (cat. no. ANT020); biotin conjugated donkey anti rabbit antibody was from Wuhan Boster Biological Technology, Ltd. (cat. no. BA1002); 3-methyladenine (3-MA) and chloroquine (CQ) were from Sigma-Aldrich; Merck KGaA; Atg16L1 small interfering RNA (siRNA) and negative control siRNA was from JTS Scientific; dextran sulfate sodium (DSS) was obtained from MP Biomedicals; the enzyme-linked immune sorbent assay (ELISA) kits for interleukin (IL)-6, IL-8 and tumor necrosis factor (TNF)- $\alpha$ were from Neobioscience Technology Co., Ltd.

Cell and bacterial strain culture. The human colonic adenoma cell line, Caco-2, was purchased from the American Type Culture Collection (ATCC) and cultured in RPMI-1640 (Gibco; Thermo Fisher Scientific, Inc.), with 10\% fetal bovine serum (FBS, Gibco; Thermo Fisher Scientific, Inc.), 100 U/ml streptomycin/penicillin (Gibco; Thermo Fisher Scientific, Inc.) and $1 \%$ HEPES (Gibco; Thermo Fisher Scientific, Inc.) at $37^{\circ} \mathrm{C}$ under $5 \% \mathrm{CO}_{2} . F$. nucleatum (ATCC 25586) and L. rhamnosus (ATCC 11982) were purchased from ATCC and cultured by the Wuhan Research Institute of First Light Industry (Wuhan, China). The methods of bacterial pellets and conditioned medium were as previously described (19). Caco- 2 cells were treated with the supernatant of $F$. nucleatum and/or L. rhamnosus to examine the effects of F. nucleatum and L. rhamnosus $(20,21)$. 3-MA $(5 \mathrm{mM})$ and CQ $(10 \mu \mathrm{M})$ treatment for $2 \mathrm{~h}$ was used to inhibit the autophagic flux.
Animals. Male C57BL/6 mice (aged 6 weeks, weighing 20-24 g) were purchased from Beijing Huafukang Biosciences Co., Ltd. and housed under specific pathogen-free (SPF) conditions with a fixed 12 light/dark cycle at $23^{\circ} \mathrm{C}$, with free access to water and food. The animal experiments in the present study were approved by the Animal Research Ethics Committee of Tongji Medical College, Huazhong University of Science and Technology (Approval ID 2016-0057).

To investigate the role of $F$. nucleatum and L. rhamnosus in the model of acute colitis, the mice were randomly divided into 6 groups as follows: i) The control; ii) L. rhamnosus; iii) F. nucleatum; iv) DSS; v) DSS +F. nucleatum; and vi) DSS $+F$. nucleatum + L. rhamnosus groups. Each group contained 7 mice. The model of acute colitis was established by the administration of $3 \%$ (wt/vol) DSS in the drinking water for 7 days, with or without the daily gavage of $10^{9}$ CFU bacteria ( $F$. nucleatum or/and L. rhamnosus) solution in phosphate-buffered saline (PBS) for 7 days prior to DSS administration as previously described $(22,23)$. In the control and DSS groups, the same volume of PBS was administered by gavage as the vehicle control. The DSS solution was refreshed every 2 days and the leftover DSS solution was measured. The body weight of the mice, as well as stool consistency and any bleeding were examined each day. The disease activity index (DAI) was calculated as follows: For weight loss: 0, no loss; $1,1-5 \% ; 2,5-10 \% ; 3,10-20 \%$ and $4,>20 \%$; for stool consistency: 0, normal; 2, loose stool; 4, diarrhea; and for stool bleeding: 0, no blood; 2, presence; and 4, gross blood (24). The mice were sacrificed on day 8 (mice which exhibited weight loss $>20 \%$ were euthanized immediately), and tissues and blood were collected for subsequent analysis. Mice were anesthetized by an intraperitoneal injection of $50 \mathrm{mg} / \mathrm{kg}$ pentobarbital and $250 \mathrm{mg} / \mathrm{kg}$ for euthanasia (25).

$R N A$ extraction and reverse transcription-quantitative $P C R$ $(R T-q P C R)$. Total RNA was extracted from the collected cells or colonic tissues using TRIzol reagent (Life Technologies; Thermo Fisher Scientific, Inc.) according to the manufacturer's instructions and reverse transcribed into complementary DNA using Prime Script RT Master Mix (Takara Biotechnology, Inc.). qPCR was performed using the LightCycler ${ }^{\circledR} 480$ SYBR I Master Mix (Roche Diagnostics), running on a Roche LightCycle R480 system (Roche Diagnostics). Gene expression was normalized relative to GAPDH and calculated using the $2^{-\Delta \Delta \mathrm{Cq}}$ method (26). The primer sequences are presented in Table I.

Western blot analysis. Proteins were extracted from the cells or tissues using RIPA lysis buffer (Beyotime Institute of Biotechnology, Inc.) supplemented with phenylmethyl sulfonyl fluoride (PMSF) protease inhibitor and phosphatase inhibitor. The total protein content in the supernatant were measured by bicinchoninic acid (BCA) assay (Thermo Fisher Scientific, Inc.). A total of $40 \mu \mathrm{g}$ cellular proteins and $80 \mu \mathrm{g}$ tissue proteins per lane were separated by 12 or $8 \%$ sodium dodecyl sulfate polyacrylamide gel electrophoresis (SDS-PAGE), and then transferred to a PVDF membrane (Millipore Corp.). This was followed by blocking with $5 \%$ non-fat milk or $5 \%$ bovine serum albumin (BSA) prior to incubation overnight at $4^{\circ} \mathrm{C}$ with primary antibodies for GAPDH, LC3B, SQSTM1/p62, 
Table I. Sequences of primers used for RT-qPCR.

\begin{tabular}{lll}
\hline Gene & \multicolumn{1}{c}{ Forward primer (5'-3') } & \multicolumn{1}{c}{ Reverse primer $\left(5^{\prime}-3^{\prime}\right)$} \\
\hline Human genes & & \\
IL-6 & ATGAGGAGACTTGCCTGGTG & GGCATTTGTGGTTGGGTCAG \\
IL-8 & CACTGCGCCAACACAGAAAT & AACTTCTCCACAACCCTCTGC \\
TNF- $\alpha$ & TACTCCCAGGTCCTCTTCAAGG & TTGATGGCAGAGAGGAGGTTG \\
GAPDH & ACCCACTCCTCCACCTTTGA & AAAGTGGTCGTTGAGGGCAA \\
Mouse genes & & \\
IL-1 $\beta$ & CTGAACTCAACTGTGAAATGCC & CTTGTTGATGTGCTGCTGCG \\
IL-6 & ACAAAGCCAGAGTCCTTCAGAG & CCACTCCTTCTGTGACTCCA \\
TNF- $\alpha$ & ACCCTCACACTCACAAACCAC & TAGCAAATCGGCTGACGGTG \\
IFN- $\gamma$ & CAGCAACAGCAAGGCGAAA & TTGAATGCTTGGCGCTGGAC \\
Beclin 1 & AGGAGCTGGAAGATGTGGAAA & CACTATACTCCCGCTGGTACTGA \\
Atg7 & ATGACCGCATGAATGAGCCT & GGTGAATCCTTCTCGCTCGT \\
Atg16L1 & GACCTCAGACCACACAGAAGA & TCCTGGCAGCATCAGAAGAATGA \\
GAPDH & CATGGCCTTCCGTGTTCCTA & TACTTGGCAGGTTTCTCCAGG \\
\hline
\end{tabular}

IL, interleukin; TNF- $\alpha$, tumor necrosis factor- $\alpha$, IFN- $\gamma$, interferon- $\gamma$; Atg16L1, autophagy-related gene 16 like 1.

Atg16L1, p-p85, p85, p-AKT, AKT, p-mTOR and mTOR (1:1,000 dilution). The membranes were then washed and incubated with goat anti rabbit secondary antibodies conjugated to $\operatorname{HRP}(1: 2,000$ dilution) for $1 \mathrm{~h}$ at room temperature the second day. Enhanced chemiluminescent reagents (Beyotime Institute of Biotechnology, Inc.) were used to detect the HRP on the immunoblots. Quantitative analysis was performed using ImageJ1 software (NIH).

ELISA. The culture supernatants of the Caco-2 cells were collected following $12 \mathrm{~h}$ of treatment with or without the supernatant of bacteria and stored at $-80^{\circ} \mathrm{C}$. The levels of IL- 8 and TNF- $\alpha$ in the supernatants were measured using respective ELISA kits according to the manufacturer's instructions, as indicated above. The absorbance was obtained at a relative nanometer wavelength using a microplate reader (Biotek Instruments, Inc.).

GFP-mCherry-LC3 plasmid transfection and confocal microscopy. Caco 2 cells at the logarithmic growth phase were seeded on coverslips at 50-70\% confluence and transfected with GFP-mCherry-LC3 plasmid DNA (Changsha Yingrun Biotechnology Co., Ltd.) using Lipofectamine 3000 transfection reagent (Invitrogen; Thermo Fisher Scientific, Inc.) according to the manufacturer's instructions and as previously described (27). The cells were transfected for $24 \mathrm{~h}$ prior to stimulation with the supernatants of $F$. nucleatum or L. rhamnosus. The cells were then washed 3 times with PBS and fixed with $4 \%$ paraformaldehyde for $15 \mathrm{~min}$. Images of red, green and merged yellow dots representing LC3 on autolysosomes and autophagosomes were captured using a confocal laser scanning microscope (Olympus Corporation). The average number of punctate fluorescent structures per cell was determined.

Transmission electron microscopy (TEM). Following stimulation with $F$. nucleatum or L. rhamnosus, the cells were fixed with $2.5 \%$ phosphate-buffered glutaraldehyde, post-fixed in $1 \%$ osmium tetroxide for $1 \mathrm{~h}$, rinsed with $0.1 \mathrm{M}$ phosphate buffer ( $\mathrm{pH}$ 7.4), and then dehydrated with increasingly graded alcohols before being embedded in Epon 812 (SPI Supplies). Ultrathin sections were cut using a ultramicrotome and observed using a FEI Tecnai G2 12TEM (FEI Company). The number of autophagic vacuoles per cell was calculated.

Atg16L1 siRNA transfection. Caco-2 cells (100,000 cells/well) were seeded in 12-well culture dishes and transfected with $100 \mathrm{nM}$ siRNA against Atg16L1 or a scrambled siRNA for negative control (NC siRNA) at $50 \%$ confluence using Lipofectamine 3000 transfection reagent (Invitrogen; Thermo Fisher Scientific, Inc.) according to the manufacturer's instructions. Gene silencing efficiency was examined by western blot analysis following $48 \mathrm{~h}$ of transfection.

Histological analysis. For histological analysis, distal colon specimens were fixed in $4 \%$ formaldehyde for $24 \mathrm{~h}$ and embedded in paraffin. The $4-\mu \mathrm{m}$-thick sections were stained with hematoxylin and eosin (H\&E) according to appropriate standard procedures (28), and then analyzed by a pathologist blinded to the identity of the data. Histological analysis was performed as previously described (29). Briefly, scores were allocated as follows: For inflammation severity: 0, none; 1, mild; 2 , moderate; 3 , severe; for the extent of inflammation: 0 , none; 1 , mucosa; 2 , mucosa and sub-mucosa; 3 , transmural; for crypt damage: 0 , none; 1 , basal $1 / 3$ damage; 2 , basal $2 / 3$ damage; 3 , crypts lost and surface epithelium present; 4 , crypts and surface epithelium loss; for percentage involvement: 0 , $0 \% ; 1,1-25 \% ; 2,26-50 \% ; 3,51-75 \% ; 4,76-100 \%$. The scores of the 4 items were added to obtain the histological score.

Immunohistochemistry (IHC). IHC was performed as previously described (30). In brief, paraffin-embedded tissues sections (4 $\mu \mathrm{m}$ thickness) were deparaffinized. Sections were 
heated in $10 \mathrm{mM}$ citrate buffer for $10 \mathrm{~min}$ for antigen-retrieval and blocked with $10 \%$ goat serum for $1 \mathrm{~h}$ at room temperature. The sections were then incubated with LC3B and p62 antibodies (1:200 dilution) overnight at $4{ }^{\circ} \mathrm{C}$ and then incubated with matched secondary antibody (1:200 dilution) for $1 \mathrm{~h}$ at room temperature the following day. The DAB kit was used to detect the signal. Images were analyzed using IHC Profiler plugin in ImageJ1 (NIH).

Statistical analysis. Each experiment was performed at least in triplicate and data are expressed as the means \pm standard error of mean (SEM). Differences between groups were performed using a two-tailed Student's t-test and one-way analysis of variance (ANOVA) followed by post-hoc Tukey's tests. Statistical analyses were performed with IBM SPSS Statistics 25 and visualized by GraphPad Prism 7.0. Differences were defined as statistically significant or highly statistically significant at $\mathrm{P}<0.05, \mathrm{P}<0.01$ and $\mathrm{P}<0.001$.

\section{Results}

L. rhamnosus alleviates DSS-induced acute colitis aggravated by $F$. nucleatum. For the in vivo experiments, preliminary experiments were performed to assess whether L. rhamnosus alone exerts any effect on the mice. As shown in Fig. S1A and B, the mice treated with L. rhamnosus alone exhibited no differences compared with the control group as regards colonic tissue destruction or pro-inflammatory cytokine expression. Therefore, the L. rhamnosus group was not taken into account in the following experiments.

Subsequently, the present study determined whether $F$. nucleatum contributes to the progression and exacerbation of IBD. F. nucleatum was administered to the mice in the model of DSS-induced acute colitis. The mice were treated with or without $F$. nucleatum or/and L. rhamnosus via gavage daily as described in the Materials and methods section for the group design at 7 days prior to the DSS administration. As shown in Fig. 1A and B, following F. nucleatum treatment, the mice presented severer complications, including hematochezia, body weight loss (three mice lost 24.2, 21.1, 22.8\% wight respectively) and a higher DAI score when compared with the mice in the DSS group $(\mathrm{P}<0.05$ and $\mathrm{P}<0.001$, respectively). These more severe phenomena were also observed evidenced by the shortening of colon length in the DSS $+F$. nucleatum group $(\mathrm{P}<0.01$, Fig. $1 \mathrm{C})$. No apparent changes in the appearance of the colon were observed between the control and F. nucleatum alone groups $(\mathrm{P}=0.436$, Fig. 1C). Subsequently, whether L. rhamnosus modifies the course of DSS-induced acute colitis induced by $F$. nucleatum was determined. As was expected, the DDS $+F$. nucleatum + L. rhamnosus group exhibited more evident positive changes than the DDS $+F$. nucleatum group as regards body weight loss, DAI score and colon length $(\mathrm{P}<0.05$, Fig. 1A-C). It was further found that compared to the mice in the DDS group, the mice in the DDS $+F$. nucleatum group exhibited more severe mucosal ulceration, inflammatory cell infiltration, crypt and gland destruction (Fig. 1D). Finally, it was found that the expression of pro-inflammatory cytokines in colon tissue, as well as the mRNA levels of IL-1 $\beta$, IL-6, TNF- $\alpha$ and interferon (IFN)- $\gamma$ were significantly decreased in DDS $+F$. nucleatum group following $L$. rhamnosus treatment (for IL-1 $\beta, \mathrm{P}<0.01$; for the other level, $\mathrm{P}<0.05$, respectively; Fig. S1C). On the whole, these results indicate that $F$. nucleatum aggravates DSS-induced acute colitis in vivo and that L. rhamnosus may induce antagonistic inflammatory functions through the downregulation of pro-inflammatory cytokine expression in mice administered DDS and $F$. nucleatum.

L. rhamnosus decreases the production of pro-inflammatory cytokines induced by $F$. nucleatum infection in vitro. To verify the pro-inflammatory effects of $F$. nucleatum in vitro, human epithelial colorectal cells $\mathrm{Caco} 2$ were treated with the supernatant of $F$. nucleatum for $12 \mathrm{~h}$ at different multiplicities of infection (MOIs). Higher gene expression levels of IL-6, IL-8 and TNF- $\alpha$ were observed in a dose-dependent manner. The results revealed a gradual increase in the levels of these cytokines along with the increasing MOI (Fig. 2A). Notably, the mRNA levels of these cytokines were significantly decreased following the addition of the supernatant of L. rhamnosus (for IL-8, $\mathrm{P}<0.0001$; for the other levels, $\mathrm{P}<0.001$; respectively; Fig. 2B). The HT29 and HCT116 cell lines were used in preliminary experiments and similar results were obtained (data not shown). However, the Caco2 cells exhibited the most significant changes in response to the stimulation. Therefore, the Caco2 cell line was selected for use the following experiments. The results of ELISA also indicated that the secretion levels of IL-6, IL- 8 and TNF- $\alpha$ in the Caco 2 cell culture medium were greater in the $F$. nucleatum group than the control group $(\mathrm{P}<0.0001$ and $\mathrm{P}<0.001$, respectively). The levels of these cytokines were significantly decreased by L. rhamnosus (all $\mathrm{P}<0.05$ respectively; Fig. $2 \mathrm{C}$ ). Collectively, these results demonstrate that $L$. rhamnosus reduces the production of pro-inflammatory cytokines induced by $F$. nucleatum infection in vitro.

L. rhamnosus restores the impaired autophagic flux induced by $F$. nucleatum in vitro. Subsequently, the present study further explored and elucidated the mechanisms through which L. rhamnosus downregulates the production of pro-inflammatory cytokines induced by $F$. nucleatum infection. Since it has been previously indicated that autophagy plays a key role in intestinal inflammation and the maintenance of intestinal homeostasis (31), the present study then detected the autophagic reaction following stimulation with F.nucleatum. The transformation of LC3-I to LC3-II is necessary for the formation of autophagosomes and is a widely accepted molecular marker for autophagy activation. Western blot analysis revealed that following exposure to $F$. nucleatum at different MOIs, a significant upregulation of LC3-II protein expression and SQSTM1/p62 expression was observed in a MOI-dependent manner. The protein levels of p62 and LC3-I also increased correspondingly, which indicated that the autophagic flux may be blocked by F. nucleatum (Fig. 3A).

In order to describe the detailed condition of the autophagic flux, CQ, a key autophagy inhibitor which can block autophagy by inhibiting the function of lysosome and then inhibit the degradation of LC3-II, was used to examine the condition of the autophagic flux. As shown in Fig. 3B, the protein level of LC3-II in the F. nucleatum + CQ group did not exhibit an obvious increase following pre-treatment with $10 \mu \mathrm{M} C \mathrm{C}$ for 

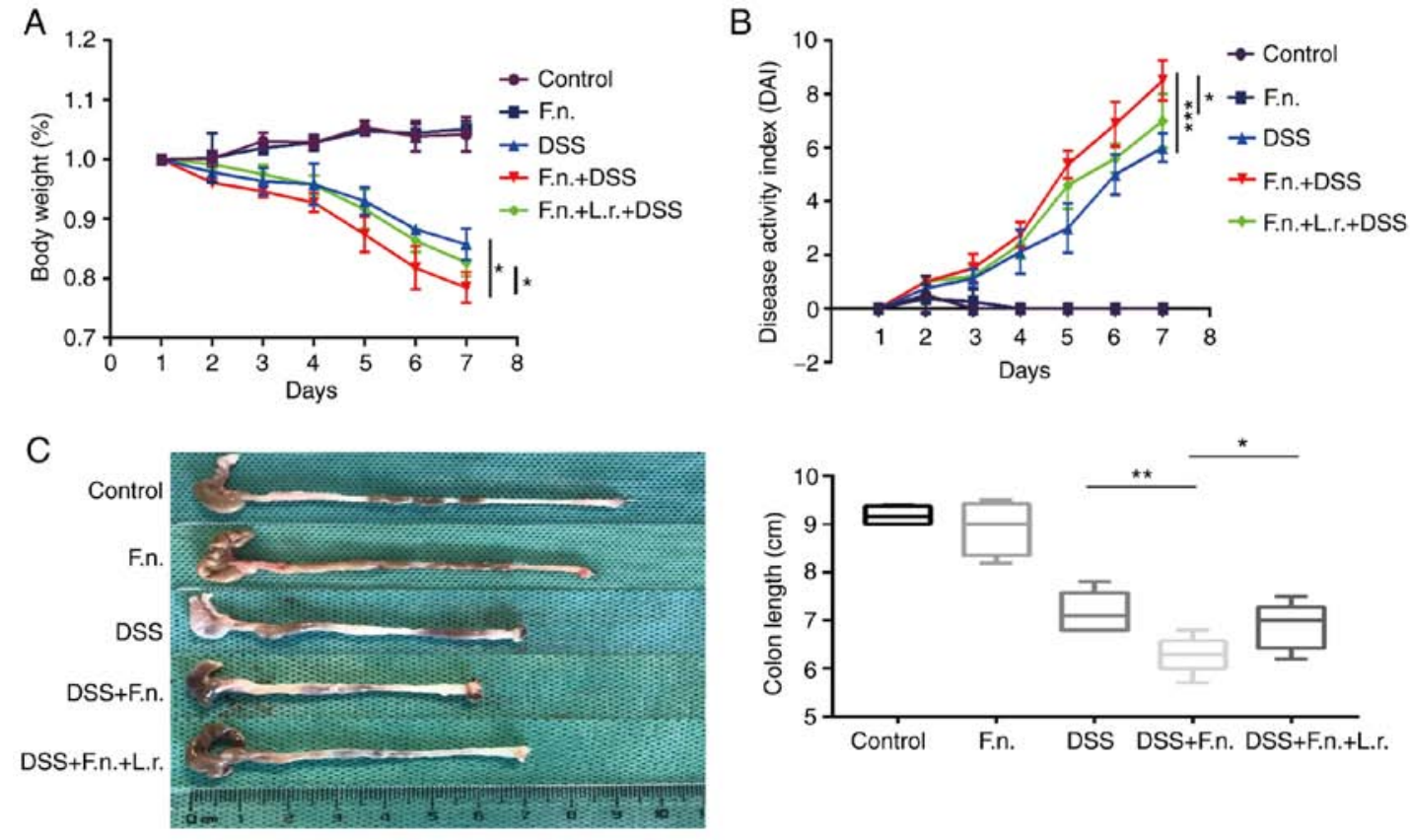

D
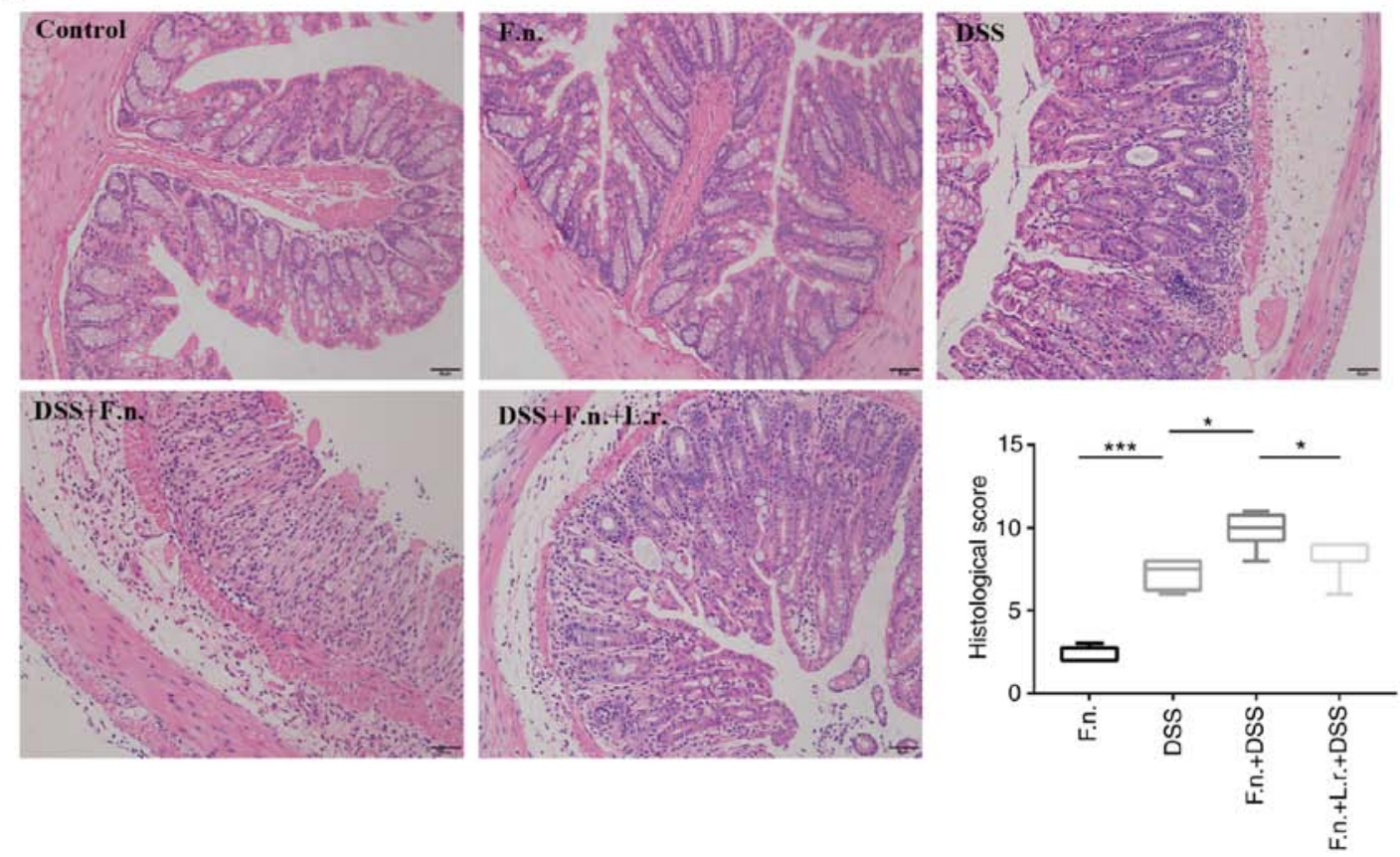

Figure 1. Lactobacillus rhamnosus alleviates DSS-induced acute colitis aggravated by $F$. nucleatum. (A and B) Body weight changes and DAI evaluation during the acute colitis process. (C) Colon length of mice in each group. (D) Serial sections from colon tissue stained with H\&E (magnification, x200) and histopathological scores of colons from mice in each group. Data are presented as the means \pm SEM of at least 3 repeated experiments. ${ }^{*} \mathrm{P} \leq 0.05,{ }^{* *} \mathrm{P} \leq 0.01$, ${ }^{* * * *} \mathrm{P}<0.001$. DSS, dextran sulfate sodium; F. nucleatum/F.n., Fusobacterium nucleatum; L.r., Lactobacillus rhamnosus; DAI, disease activity index.

$2 \mathrm{~h}$ compared to treatment with $F$. nucleatum supernatant only ( $\mathrm{P}=0.59)$, indicating that $F$. nucleatum impairs the autophagic flux process.

Subsequently, the effects of L. rhamnosus were evaluated and it was found that the ratio of LC3-II/GAPDH and p62/GAPDH was lower $(\mathrm{P}<0.05)$ following the addition of L. rhamnosus (Fig. 3C and D). Furthermore, L. rhamnosus treatment not only increased the expression of LC3B-II, but also the protein level of LC3-II in the L. rhamnosus + CQ group exhibited an obvious increase compared to the L. rhamnosus only group, indicating that L. rhamnosus promoted the autophagic process (Fig. 3E).
To further confirm the autophagic flux status, GFP-mCherry-LC3 plasmid was transfected into Caco2 cells. Following transfection for $24 \mathrm{~h}$ and treatment with the indicated interventions, confocal microscopic analysis revealed that $F$. nucleatum stimulation resulted in the accumulation of red puncta with the significant augmentation of of green puncta, suggesting that the fusion of autophagosomes and lysosomes was blocked (Fig. 4A). From electron microscopic analysis at the ultrastructural level in the F. nucleatum-treated cells, autophagosome structures were also more prevalent (Fig. 4B). When the transfected cells were treated with $L$. rhamnosus culture supernatants, the number of red 

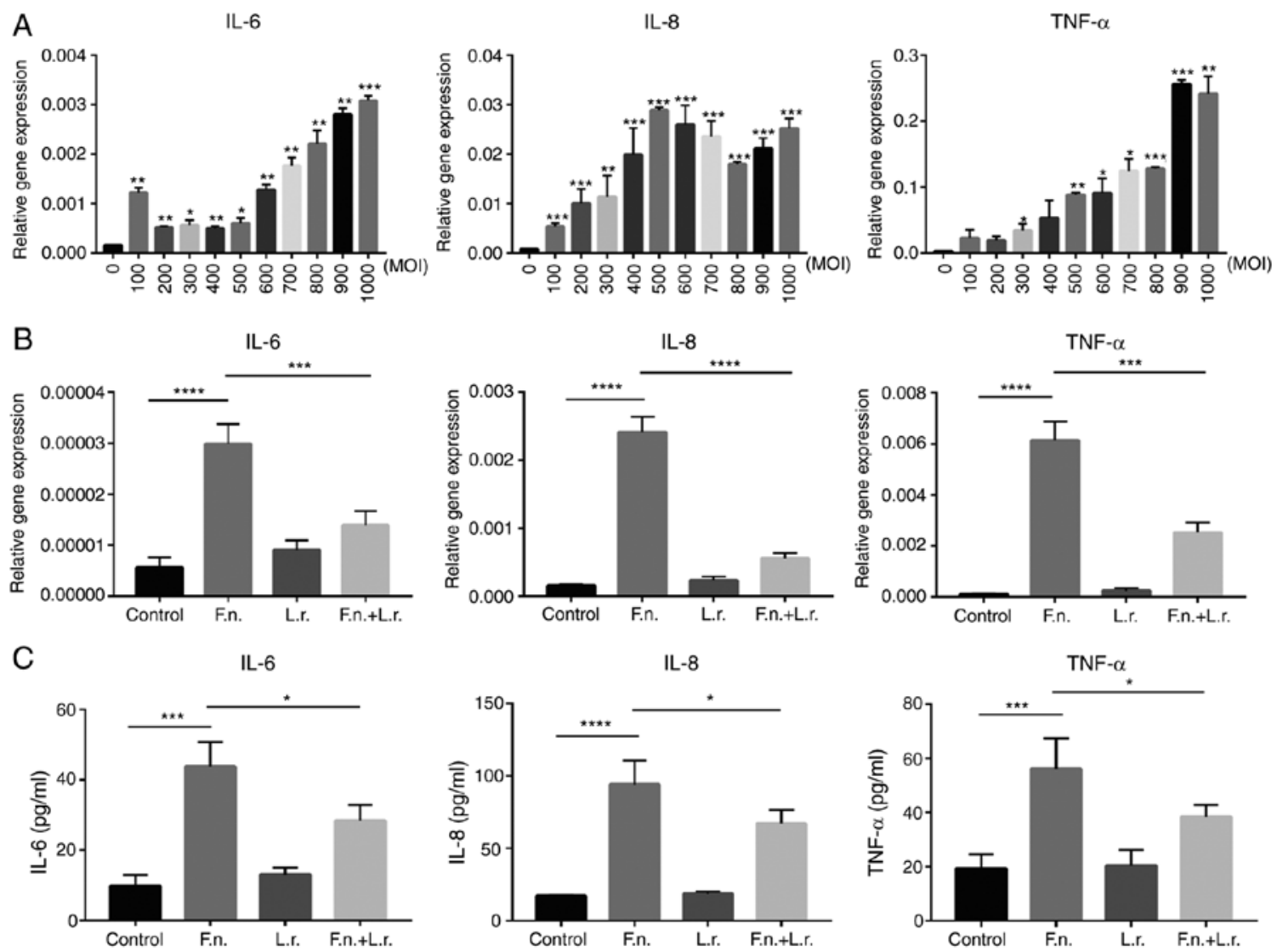

Figure 2. Lactobacillus rhamnosus decreases pro-inflammatory cytokine production induced by F. nucleatum infection in vitro. (A) The relative gene expression of IL-6, IL-8, TNF- $\alpha$ in Caco2 cells treated with $F$. nucleatum supernatant at increasing MOIs. (B) The relative gene expression of IL-6, IL-8, TNF- $\alpha$ in Caco2 cells treated with $F$. nucleatum and Lactobacillus rhamnosus supernatant. (C) The secretion of IL-6, IL-8, TNF- $\alpha$ proteins was detected by ELISA in cell culture medium. Data are presented as the means $\pm \mathrm{SEM}$ of at least 3 repeated experiments. ${ }^{*} \mathrm{P} \leq 0.05,{ }^{* * *} \mathrm{P} \leq 0.01,{ }^{* * * *} \mathrm{P}<0.001,{ }^{* * * * *} \mathrm{P}<0.0001$. F. nucleatum/F.n., Fusobacterium nucleatum; L.r., Lactobacillus rhamnosus.

\section{A}

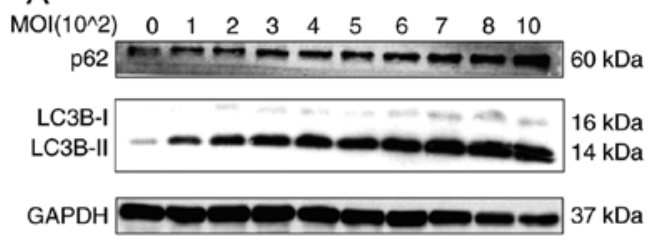

C

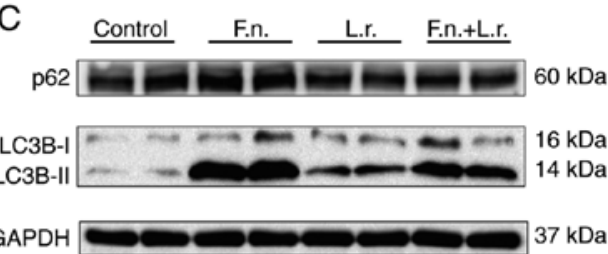

B

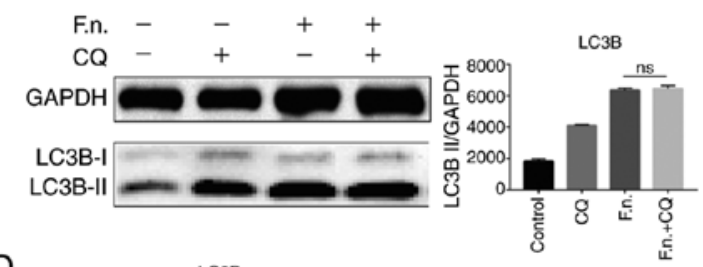

D
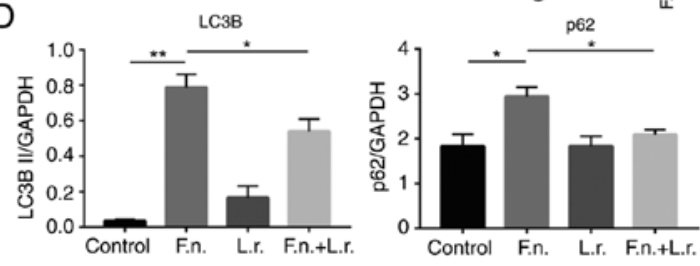
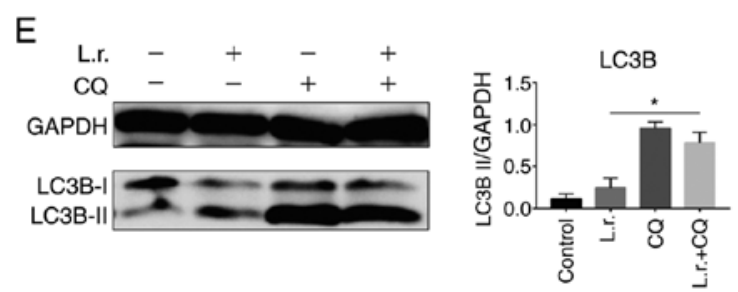

Figure 3. Lactobacillus rhamnosus restores the impaired autophagic flux induced by F. nucleatum in vitro. (A) Representative LC3B and SQSTM1/p62 western blots of Caco2 cells treated with $F$. nucleatum. supernatant at increasing MOIs for $12 \mathrm{~h}$. GAPDH was used as a loading control. (B) Caco2 cells challenged with F. nucleatum were pre-treated with or without CQ. Western blot analysis was conducted to detect the LC3B level. (C) Protein levels of LC3B and SQSTM1/p62 of Caco2 cells treated with F. nucleatum. and Lactobacillus rhamnosus supernatant for $12 \mathrm{~h}$. (D) Histogram of relative expression of LC3B and p62. (E) Caco2 cells challenged with Lactobacillus rhamnosus were pre-treated with or without CQ. Western blot analysis was conducted to detect the LC3B level. ${ }^{*} \mathrm{P} \leq 0.05,{ }^{* *} \mathrm{P} \leq 0.01$. F. nucleatum/F.n., Fusobacterium nucleatum; L.r., Lactobacillus rhamnosus; $\mathrm{CQ}$, chloroquine. 
A GFP
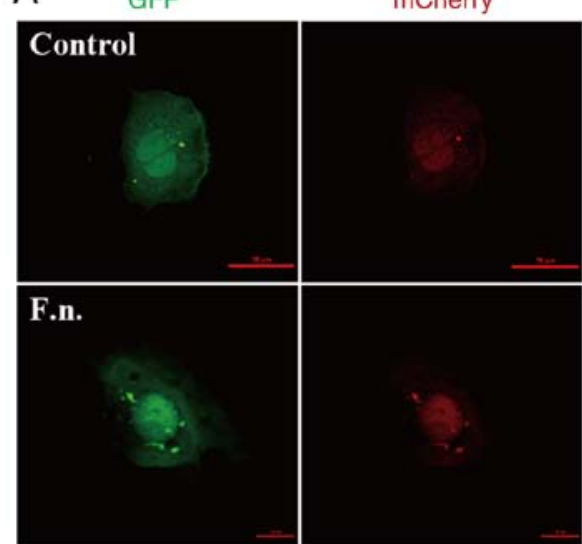

L.r.
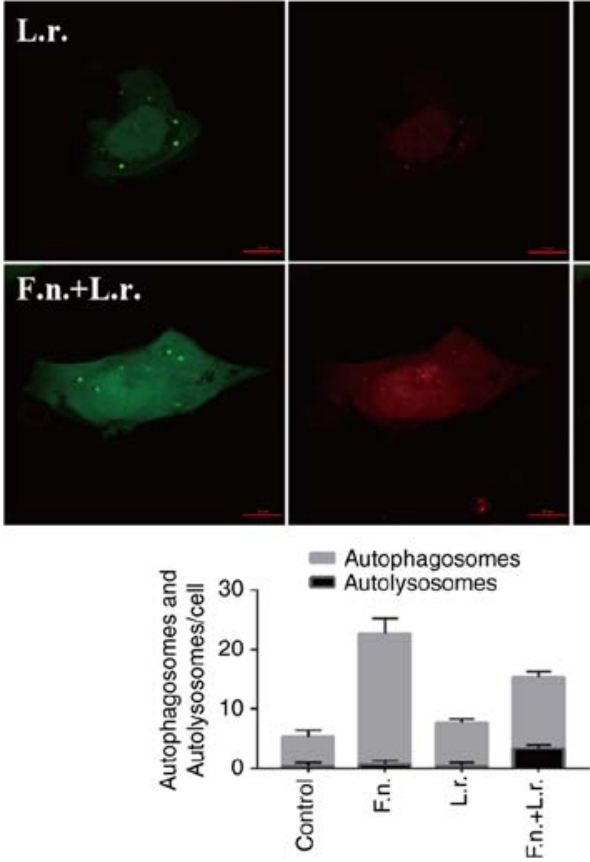

Merge
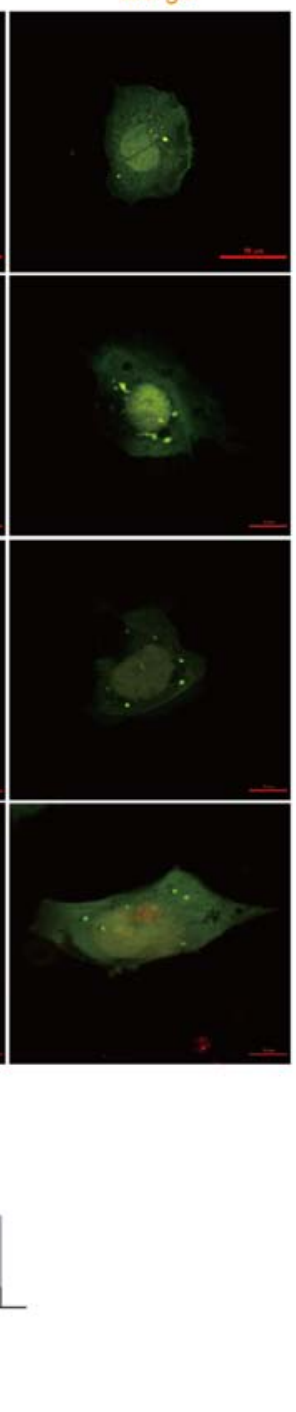

B
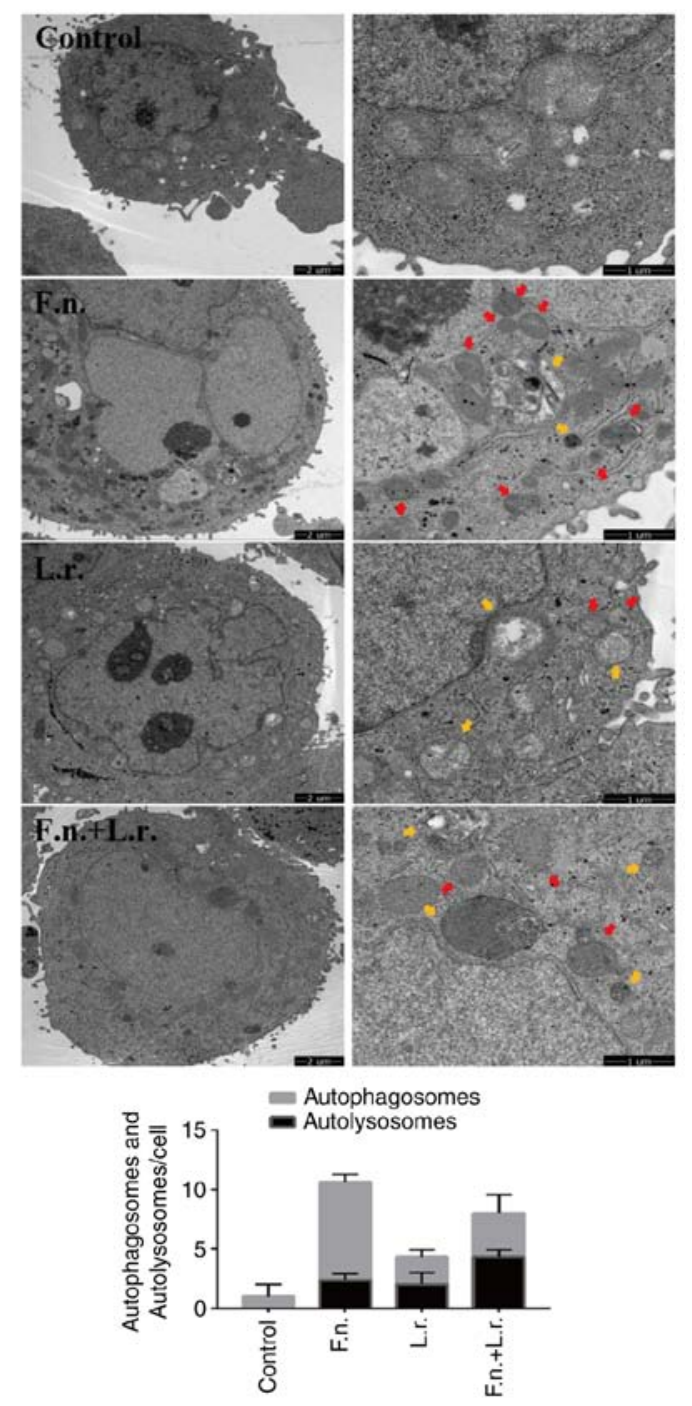

Figure 4. Identification of autophagy by immunofluorescence and TEM. (A) Confocal micrographs illustrating the accumulation of GFP-mCherry-LC3 in Caco2 cells treated with $F$. nucleatum or Lactobacillus rhamnosus (magnification, $\mathrm{x} 400$ ). (B) Ultrastructural features of autophagic vacuoles in Caco 2 cells treated with F. nucleatum or Lactobacillus rhamnosus (magnification, x1,700 and x5,000). Red arrows indicate autophagosomes and yellow arrows indicate autolysosomes. F. nucleatum/F.n., Fusobacterium nucleatum; L.r., Lactobacillus rhamnosus.

fluorescence signals markedly increased (Fig. 4A). More autolysosomes were also observed in the F. nucleatum group when exposed to the L. rhamnosus supernatants (Fig. 4B). Collectively, these results demonstrate that in the Caco2 cells, L. rhamnosus probiotics can regulate and restore the impaired autophagic flux associated with $F$. nucleatum stimulation.

L. rhamnosus increases autophagy inhibited by F. nucleatum infection in vivo. To assess whether the administration of $F$. nucleatum alters the course of autophagy in the progression of colitis, experiments were conducted with the mice from the model of DSS-induced colitis. Western blot analysis was performed to examine the ratio of LC3-II/LC3-I and p62/GAPDH. As shown in Fig. 5A, pre-treatment of the mice with $F$. nucleatum was associated with a decreased ratio of LC3-II/LC3-I (although LC3-II expression increased, the increase in LC3-I expression was relatively more evident compared to the other groups) and an increased p62/GAPDH ratio in response to DSS administration (all $\mathrm{P}<0.05$ ). The ratio was reversed when the mice in the $F$. nucleatum + DSS group were treated with L. rhamnosus $(\mathrm{P}<0.01$ and $\mathrm{P}<0.05$, respectively). Consistent with the above-mentioned data, the IHC analysis of LC3B and p62 expression in the colon tissues further confirmed the antagonistic effects of $F$. nucleatum and L. rhamnosus (Figs. 5B and S2A). Following L. rhamnosus intervention, F. nucleatum-DSS impaired autophagy was significantly restored.

In addition, the mRNA levels of Beclin1, Atg7 and Atg16L1 were also measured, which are key genes associated with autophagy. The levels of Beclin1, Atg7 and Atg16L1 were significantly lower in the mice that were administered $F$. nucleatum and DSS compared to those administered F. nucleatum or DSS alone $(\mathrm{P}<0.05,<0.01$ and $<0.01$, respectively; Fig. S2B) and were higher in response to L. rhamnosus treatment (all $\mathrm{P}<0.05)$. Collectively, L. rhamnosus significantly attenuated the F. nucleatum-induced intestinal blocking of autophagy in vivo. 


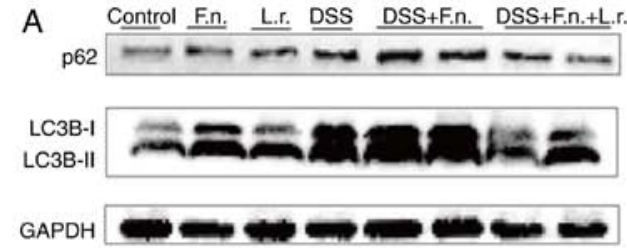

B
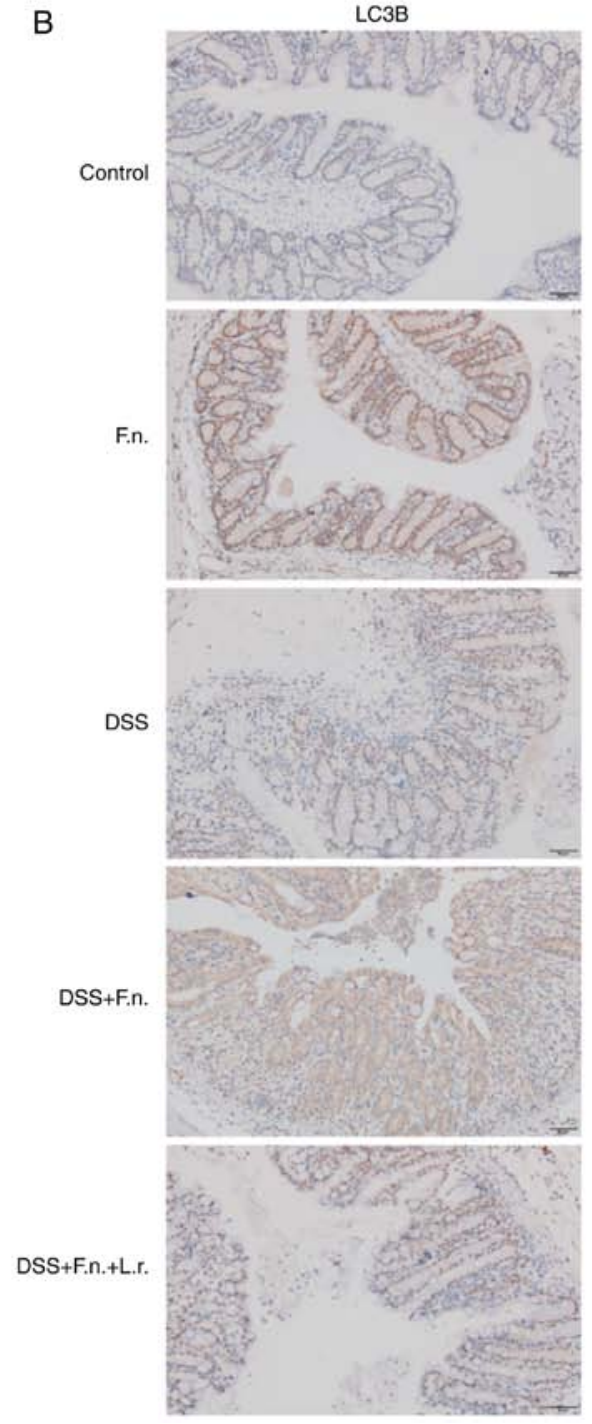
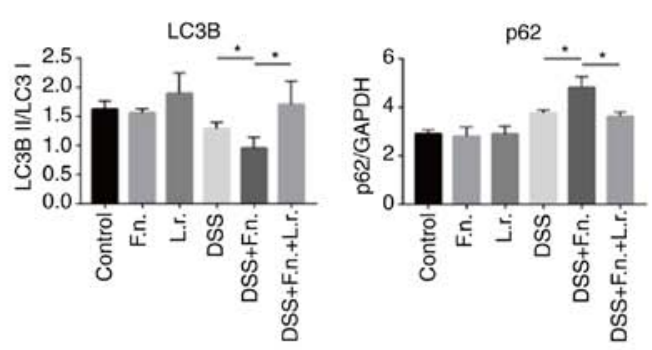

p62

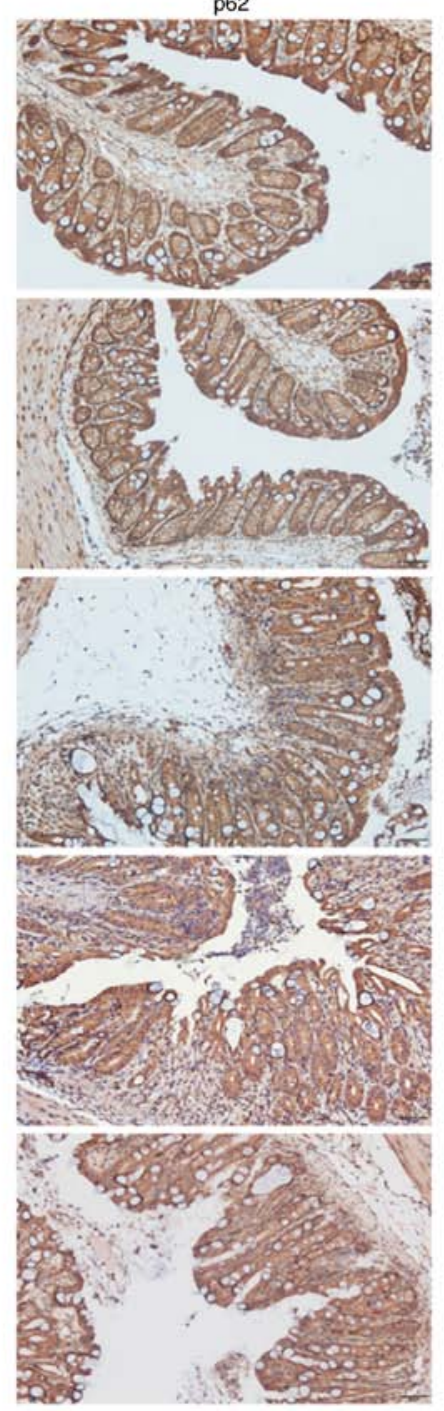

Figure 5. Lactobacillus rhamnosus increases autophagy inhibited by F. nucleatum infection in vivo. (A) Representative western blot bands for LC3B proteins in colon tissues. GAPDH was used as a loading control. (B) Representative images showing LC3B and SQSTM1/p62 immunostaining (magnification, x200). Data are presented as the means $\pm \mathrm{SEM}$ of at least 3 repeated experiments. ${ }^{*} \mathrm{P} \leq 0.05$. F. nucleatum/F.n., Fusobacterium nucleatum; L.r., Lactobacillus rhamnosus.

L. rhamnosus restores the impaired autophagic flux via the AKT/mTOR pathway. The AKT/mTOR pathway has been shown to be a key regulator of the autophagic process. The mTOR complex 1 (mTORC1) is a major negative regulator of autophagy, while AKT is a major upstream modulator of mTORC1 (32). The present study therefore assessed AKT and mTOR expression in the current experimental system in order to confirm whether $F$. nucleatum and L. rhamnosus regulate autophagy through this pathway. It was found that the protein levels of p-mTOR and p-AKT gradually increased to a peak in a concentration-depend manner following exposure to F. nucletum and then gradually decreased (Fig. 6A and B).
As was expected, the F. nucleatum group exhibited higher p-mTOR, p-p85 and p-AKT protein expression levels compared with the control or L. rhamnosus only groups. L. rhamnosus treatment induced a decrease in these protein levels and enhanced autophagy (for $\mathrm{p}-\mathrm{p} 85, \mathrm{P}<0.01$; for the other levels, $\mathrm{P}<0.05$; Figs. $6 \mathrm{C}$ and S3A). Consistent with these findings, the results obtained from the colonic tissues were similar to those obtained with the cell experiments (Figs. 6D and S3B). The mice in the $F$. nucleatum + DSS group exhibited a decreased p-mTOR and p-AKT protein level following L. rhamnosus intervention, although the p-p85 levels were not significantly altered. All these results demonstrated that the AKT/mTOR 
A

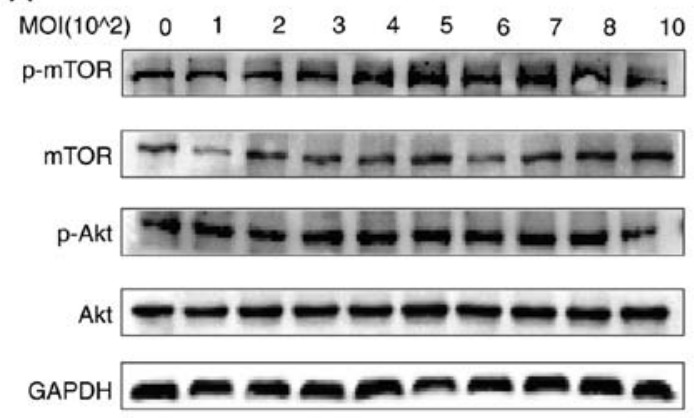

C

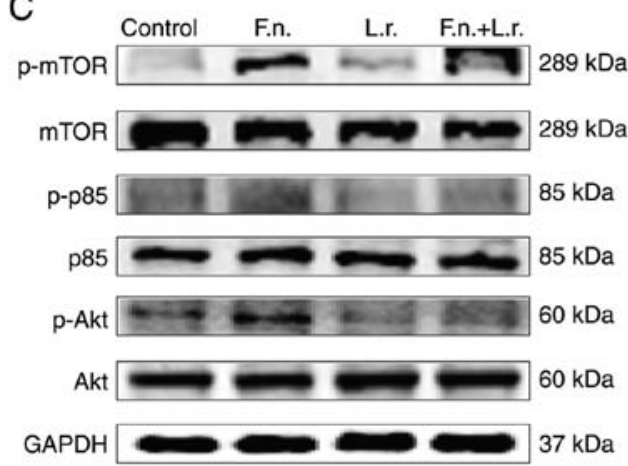

B
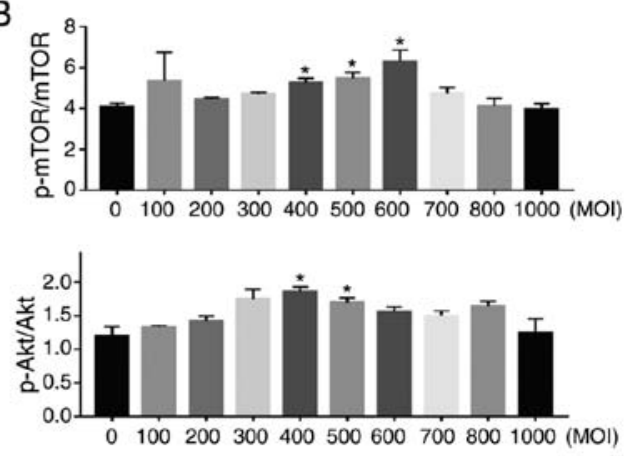

D

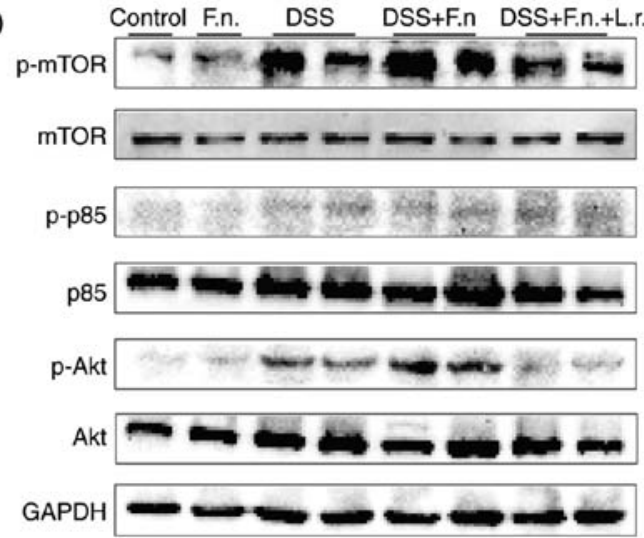

Figure 6. Lactobacillus rhamnosus restores the impaired autophagic flux via AKT/mTOR pathway. (A and B) Effects of increasing MOI $F$. nucleatum on p-mTOR, mTOR, p-AKT and AKT protein levels in Caco2 cells are indicated by representative western blots. (C) Western blot analysis of p-mTOR, mTOR, p-p85, p85, p-AKT and AKT levels in Caco2 cells, respectively. (D) Western blot analysis of p-mTOR, mTOR, p-p85, p85, p-AKT and AKT levels in Caco2 cells and colon tissues, respectively. ${ }^{*} \mathrm{P} \leq 0.05$. F. nucleatum/F.n., Fusobacterium nucleatum; L.r., Lactobacillus rhamnosus; DSS, dextran sulfate sodium.

pathway was involved in the regulation of the autophagy process and that L. rhamnosus attenuated intestinal colitis aggravated by $F$. nucleatum relative.

Blocking the autophagic flux reinforces the production of pro-inflammatory cytokines induced by F. nucleatum and partly inhibits the effects of Lactobacillus rhamnosus. As illustrated above, F. nucleatum intensified colonic inflammation in vitro and in vivo; the mechanisms involved the blocking of the autophagic flux. To further investigate the phenomenon of the autophagic flux, 2 autophagy chemical inhibitors of autophagy, 3-MA and CQ, were used prior to the $F$. nucleatum or L. rhamnosus treatment of the Caco2 cells. As shown in Fig. 7, the autophagy inhibitors significantly enhanced the gene expression levels of IL-6, IL- 8 and TNF- $\alpha$ (for IL-8, $\mathrm{P}<0.01$; for the other levels, $\mathrm{P}<0.05$, respectively). As was expected, L. rhamnosus did not markedly reduce the mRNA levels of these pro-inflammatory cytokines following pre-treatment with the autophagy inhibitors (for IL-8 and TNF- $\alpha$ in CQ treatment, $\mathrm{P}<0.01$; for the other levels, $\mathrm{P}<0.05$, respectively).

Subsequently, the present study examined the L. rhamnosus-mediated activation of the F. nucleatum-induced autophagic flux damage using gene interference. Caco2 cells were transfected with siRNA designed to inhibit Atg16LI expression and this was expected to attenuate the mediating effects of $L$.rhamnosus on the $F$. nucleatum-induced blocking of autophagy. The knockdown of Atg16L1 expression by siRNA effectively decreased its expression by $66.8 \%$ compared to the untransfected cells (Fig. 8A). The cytokine production of the siRNA-transfected cells was subsequently examined in response to $F$. nucleatum and L. rhamnosus treatment using ELISA and RT-qPCR. Consistent with the above-mentioned results, IL- 8 and TNF-a production induced by $F$. nucleatum treatment increased in the presence of Atg16L1 siRNA and the effects of L. rhamnosus were suppressed (Fig. 8B). The results of RT-qPCR revealed a similar variation tendency for the mRNA levels (Fig. 8C). Taken together, the mediation of autophagy by the probiotics L. rhamnosus was involved in the protective effects against F. nucleatum-related intestinal inflammation.

\section{Discussion}

There is increasing evidence of the connection between intestinal bacterium and defective autophagy in the pathogenesis of IBD. The present study demonstrated that L. rhamnosus attenuated $F$. nucleatum-related intestinal inflammation by mediating the autophagy of intestinal epithelial cells. First, establishing an in vitro and in vivo model revealed that L. rhamnosus effectively alleviated colitis and attenuated the production of pro-inflammation cytokines aggravated by $F$. nucleatum. Subsequently, the status of autophagy was detected and it was found that L. rhamnosus restored the autophagic flux impaired by $F$. nucleatum in vitro and in vivo. Moreover, the PI3K/AKT/mTOR pathway was investigated and it was demonstrated that it was involved in the autophagic process. Finally, autophagy was interfered with 

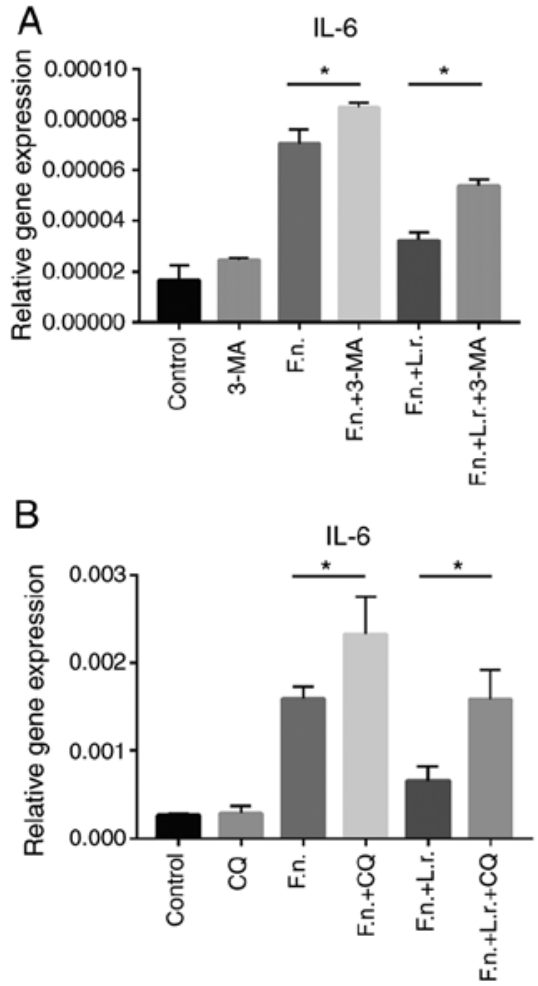
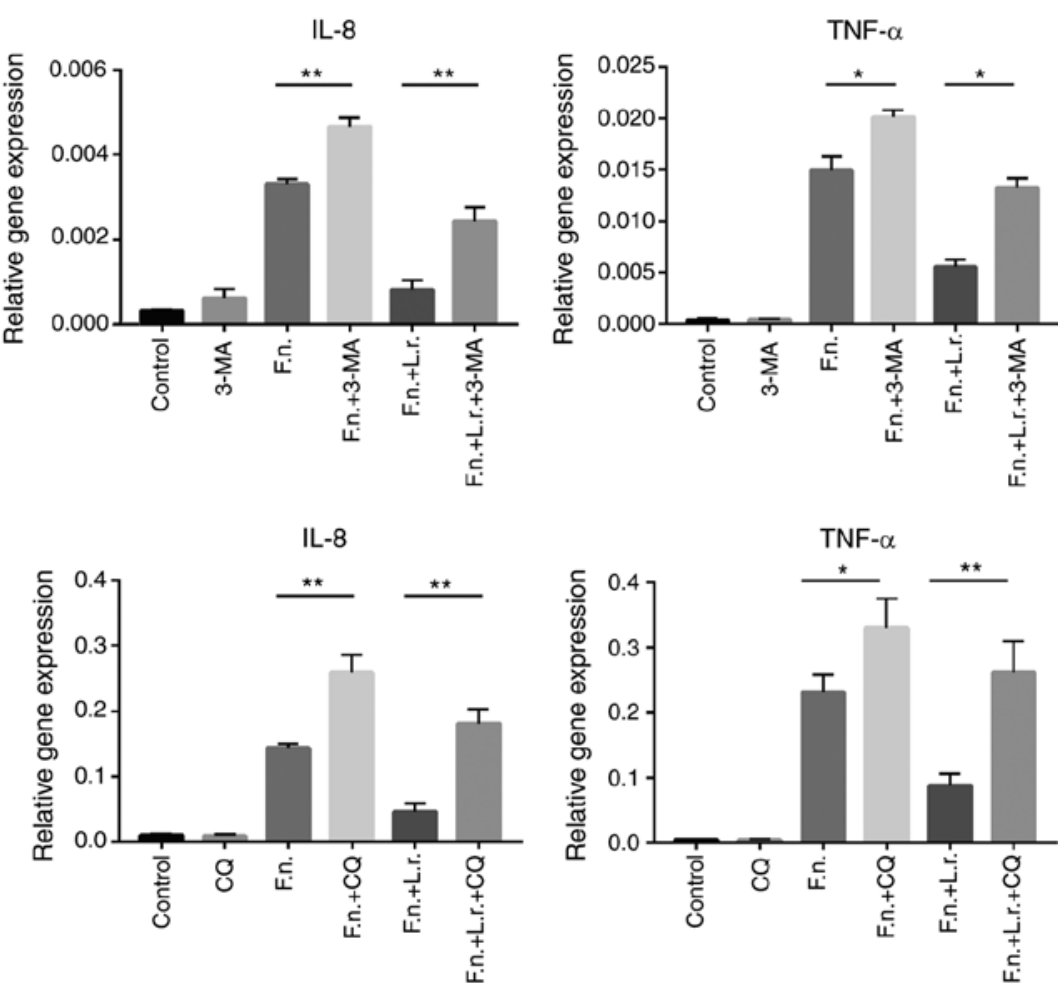

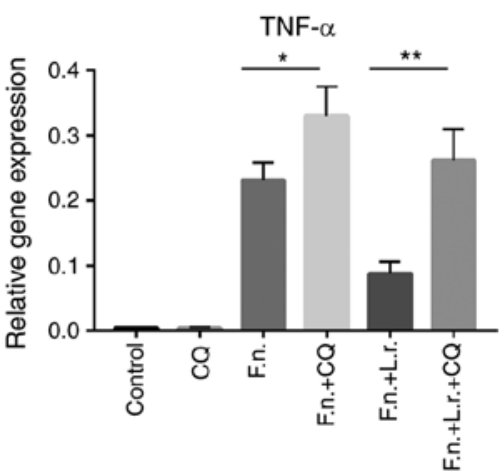

Figure 7. Blocking of the autophagic flux with autophagy inhibitors reinforces pro-inflammatory cytokine production induced by $F$. nucleatum and partly inhibits the effects of Lactobacillus rhamnosus. (A and B) Caco2 cells were pre-treated with 3-MA or CQ before being challenged with F. nucleatum or Lactobacillus rhamnosus. RT-qPCR were performed to exam the mRNA levels of IL-6, IL-8 and TNF- $\alpha .{ }^{~} \mathrm{P} \leq 0.05,{ }^{* *} \mathrm{P} \leq 0.01$. F. nucleatum/F.n., Fusobacterium nucleatum; L.r., Lactobacillus rhamnosus; CQ, chloroquine; 3-MA, 3-methyladenine.

A
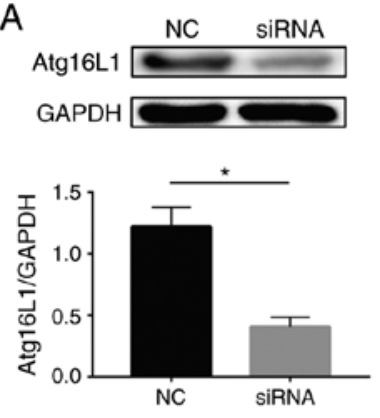

C

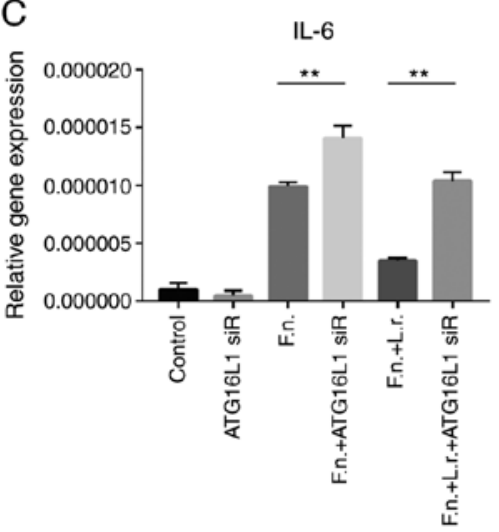

B
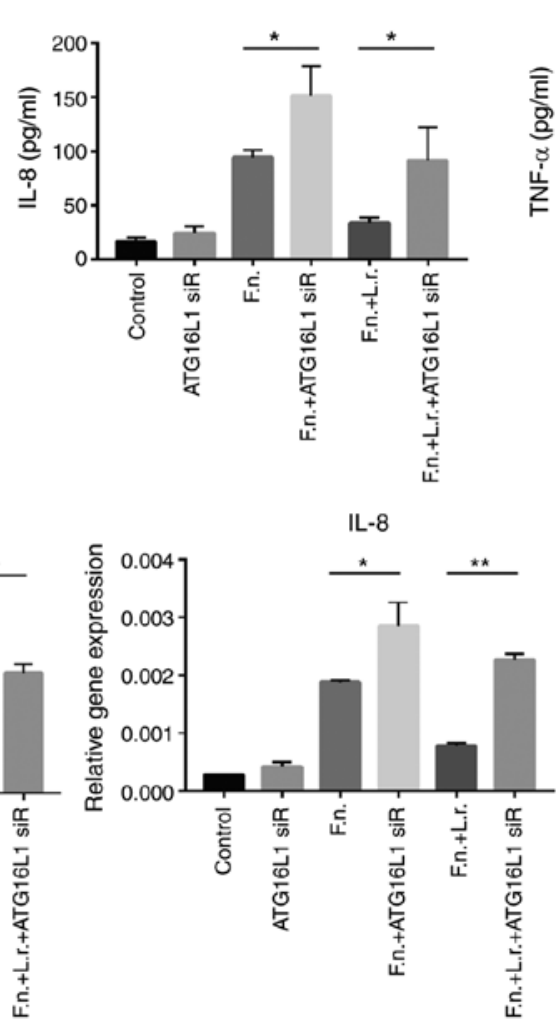
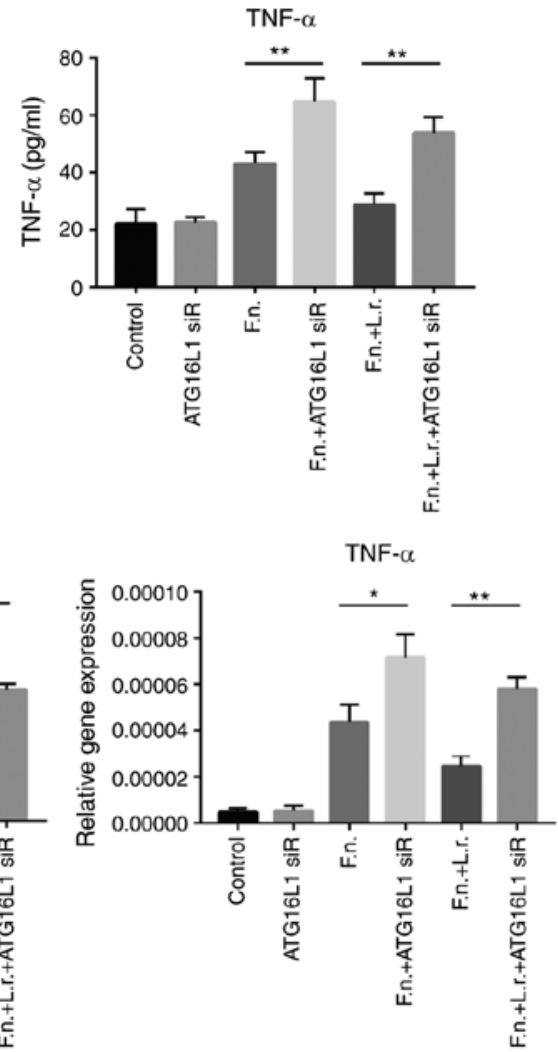

Figure 8. Blocking autophagic flux with RNA interference reinforces pro-inflammatory cytokine production induced by $F$. nucleatum and partly inhibits the effects of Lactobacillus rhamnosus on autophagy. (A) Western blot analysis verification of Atg16L1 siRNA efficiency. (B and C) Caco2 cells were transfected with Atg16L1 siRNA for $48 \mathrm{~h}$ following treatment with F. nucleatm or Lactobacillus rhamnosus. Protein levels of IL-8 and TNF- $\alpha$ in cell culture medium detected by ELISA and mRNA levels of IL-6, IL-8 and TNF- $\alpha$ were analyzed by RT-qPCR. Data are presented as the means \pm SEM of at least 3 repeated experiments. ${ }^{*} \mathrm{P} \leq 0.05,{ }^{* *} \mathrm{P} \leq 0.01$. F. nucleatum/F.n., Fusobacterium nucleatum; L.r., Lactobacillus rhamnosus. 
using autophagy inhibitors and siRNAs in Caco2 cells. As a result, the pro-inflammatory effects of $F$. nucleatum were enhanced and the anti-inflammatory of effects L. rhamnosus were impaired.

Previous studies have demonstrated the association of $F$. nucleatum with intestinal inflammation, as it can generate a pro-inflammatory microenvironment (33). Moreover, it is easier to isolate Fusobacterium spp. from patients with intestinal inflammatory disease compared with healthy controls, more than a half of which are $F$. nucleatum and these strains are significantly more invasive than those from healthy controls (6). Another study found that $F$. nucleatum regulated M1 macrophage polarization and the secretion of IFN- $\gamma$ by Th1-related cytokines, thus promoting the progression of ulcerative colitis (22). Consistent with these findings, the present study found that $F$. nucleatum intensified the severity of experimental colitis. However, the underlying mechanisms remain unknown.

It is known that probiotics can improve the intestinal mucosal barrier by promoting the secretion of anti-inflammatory factors and inhibiting the growth of harmful bacteria in the intestine (34). L. rhamnosus is a widely used probiotic with the ability to restrain pathogenic bacteria. It has been reported L. rhamnosus can produce factors capable of suppressing Clostridium difficile-induced inflamed production (35) and attenuate enterohemorrhagic Escherichia coli-induced changes in paracellular permeability in epithelial cell monolayers, thus protecting epithelial barrier function (36). However, to the best of our knowledge, no study to date has reported the effects of L. rhamnosus on F. nucleatum. In the present study, it was found that L. rhamnosus attenuated DSS-induced colitis aggravated by $F$. nucleatum, as shown by an improvement in pathological features, DAI scores, colon length and pro-inflammatory cytokine expression. Similarly, L. rhamnosus decreased F. nucleatum-induced pro-inflammatory cytokine production in Caco 2 cells. However, there are some limitations to the present study. A group administered DSS + L. rhamnosus mice may be useful to further support the findings, since it could clarify the repairing effect of L. rhamnosus on DSS-induced colitis without $F$. nucleatum. However, it may still be difficult to clarify whether L. rhamnosus purely acts on DSS or on F. nucleatum as well. Therefore, cell experiments were also performed to further clarify whether L. rhamnosus can protect intestinal epithelial cells from $F$. nucleatum infection without other influencing factors.

The present study then focused on the underlying mechanisms. Autophagy plays a vital role in host defenses against microbial infection and protects the intestine from injury in the process of IBD (37). With the conditional knockdown of crucial autophagy-related genes in mice or colonic epithelial cells, the susceptibility to DSS-induced colitis is enhanced and an abnormal microflora is formed (12). Crohn's disease-associated adherent invasive Escherichia coli (AIEC) can reduce the levels of Atg5 and Atg16L1 and inhibit autophagy, thus increasing the inflammatory response (38). On the other hand, probiotics can also activate autophagy to eliminate intracellular bacteria. Thus, it was hypothesized that autophagy may be involved in the process of F. nucleatum invasion and L. rhamnosus protection. In the present study, it was demonstrated that autophagy was deregulated following the administration of $F$. nucleatum in vitro and in vivo, while L. rhamnosus restored the autophagic flux.
Autophagy interference experiments revealed that the inhibition of autophagy increased the secretion of pro-inflammatory cytokines induced by $F$. nucleatum and suppressed the 'anti-F. nucleatum' effects of L. rhamnosus. Therefore, it is reasonable to point out that autophagy is involved in the said circumstances. However, although autophagy inhibitors or siRNA weakened the protective effects of L. rhamnosus on F. nucleatum, these effects were not completely inhibited. This may indicate that there are other inhibitory and mediating effects of L. rhamnosus on the actions of $F$. nucleatum, apart from the restoration of autophagy. Thus, further studies are warranted to fully investigate this matter. Furthermore, the present study examined the changes in the $\mathrm{PI} 3 \mathrm{~K} / \mathrm{AKT} / \mathrm{mTOR}$ pathway, as it serves as one of the main regulatory pathways of autophagy (32). It was found that the changes in this pathway conformed to the changes in autophagy.

In conclusion, the present study demonstrates that $L$. rhamnosus plays a protective role in the pathogenesis of $F$. nucleatum-related colitis and that the mediation of autophagy is involved in this process. The findings of the present study may provide new insight into the pathogenesis and therapy of IBD.

\section{Acknowledgements}

Not applicable.

\section{Funding}

The present study was financially supported by the National Natural Science Foundation of China (grant nos. 81800467, 81330014, 81720108006 and 81974062).

\section{Availability of data and materials}

The datasets used and analyzed during the current study are available from the corresponding author on reasonable request.

\section{Authors' contributions}

CD performed the experiments, analyzed the data and drafted the manuscript. XT, WW and WQ were involved in the evaluation of the data. XF and XD were involved in the culture of the bacteria. SZ assisted in the animal experiments. $\mathrm{CH}$ and $\mathrm{XH}$ designed the study, revised the manuscript, provided funding and obtained grants. All authors read and approved the final manuscript.

\section{Ethics approval and consent to participate}

The animal experiments in the present study were approved by the Animal Research Ethics Committee of Tongji Medical College, Huazhong University of Science and Technology (approval ID 2016-0057).

\section{Patient consent for publication}

Not applicable.

\section{Competing interests}

The authors declare that they have no competing interests. 


\section{References}

1. Kaser A, Zeissig S and Blumberg RS: Inflammatory bowel disease. Annu Rev Immunol 28: 573-621, 2010.

2. Olivera P, Danese S, Jay N, Natoli G and Peyrin-Biroulet L: Big data in IBD: A look into the future. Nat Rev Gastroenterol Hepatol 16: 312-321, 2019.

3. Mazmanian SK, Round JL and Kasper DL: A microbial symbiosis factor prevents intestinal inflammatory disease. Nature 453: 620-625, 2008

4. Garrett WS, Gallini CA, Yatsunenko T, Michaud M, DuBois A, Delaney ML, Punit S, Karlsson M, Bry L, Glickman JN, et al: Enterobacteriaceae act in concert with the gut microbiota to induce spontaneous and maternally transmitted colitis. Cell Host Microbe 8: 292-300, 2010.

5. Chu H, Khosravi A, Kusumawardhani IP, Kwon AH, Vasconcelos AC, Cunha LD, Mayer AE, Shen Y, Wu WL, Kambal A, et al Gene-microbiota interactions contribute to the pathogenesis of inflammatory bowel disease. Science 352: 1116-1120, 2016.

6. Strauss J, Kaplan GG, Beck PL, Rioux K, Panaccione R, Devinney R, Lynch T and Allen-Vercoe E: Invasive potential of gut mucosa-derived Fusobacterium nucleatum positively correlates with IBD status of the host. Inflamm Bowel Dis 17: 1971-1978, 2011

7. Klionsky DJ: Autophagy: From phenomenology to molecular understanding in less than a decade. Nat Rev Mol Cell Biol 8: 931-937, 2007.

8. Lévy J, Cacheux W, Bara MA, L'Hermitte A, Lepage P, Fraudeau M, Trentesaux C, Lemarchand J, Durand A, Crain AM, et al: Intestinal inhibition of Atg7 prevents tumour initiation through a microbiome-influenced immune response and suppresses tumour growth. Nat Cell Biol 17: 1062-1073, 2015.

9. Narendra D, Tanaka A, Suen DF and Youle RJ: Parkin is recruited selectively to impaired mitochondria and promotes their autophagy. J Cell Biol 183: 795-803, 2008.

10. Mialet-Perez J and Vindis C: Autophagy in health and disease: Focus on the cardiovascular system. Essays Biochem 61: 721-732, 2017.

11. Chai $\mathrm{P}, \mathrm{Ni} \mathrm{H}$, Zhang $\mathrm{H}$ and Fan $\mathrm{X}$ : The evolving functions of autophagy in Ocular health: A double-edged sword. Int J Biol Sci 12: 1332-1340, 2016.

12. Tsuboi K, Nishitani M, Takakura A, Imai Y, Komatsu M and Kawashima H: Autophagy protects against colitis by the maintenance of normal gut microflora and secretion of mucus. J Biol Chem 290: 20511-20526, 2015.

13. Benjamin JL, Sumpter R Jr, Levine B and Hooper LV: Intestinal epithelial autophagy is essential for host defense against invasive bacteria. Cell Host Microbe 13: 723-734, 2013.

14. Sanders ME, Guarner F, Guerrant R, Holt PR, Quigley EM, Sartor RB, Sherman PM and Mayer EA: An update on the use and investigation of probiotics in health and disease. Gut 62: 787-796, 2013

15. Gionchetti P, Lammers KM, Rizzello F and Campieri M: Probiotics and barrier function in colitis. Gut 54: 898-900, 2005.

16. Ng SC, Plamondon S, Kamm MA, Hart AL, Al-Hassi HO, Guenther T, Stagg AJ and Knight SC: Immunosuppressive effects via human intestinal dendritic cells of probiotic bacteria and steroids in the treatment of acute ulcerative colitis. Inflamm Bowel Dis 16: 1286-1298, 2010.

17. Claes IJ, Lebeer S, Shen C, Verhoeven TL, Dilissen E, De Hertogh G, Bullens DM, Ceuppens JL, Van Assche G, Vermeire $S$, et al: Impact of lipoteichoic acid modification on the performance of the probiotic Lactobacillus rhamnosus GG in experimental colitis. Clin Exp Immunol 162: 306-314, 2010.

18. Plaza-Díaz J, Ruiz-Ojeda FJ, Vilchez-Padial LM and Gil A: Evidence of the anti-inflammatory effects of probiotics and synbiotics in intestinal chronic diseases. Nutrients 9: 555, 2017.

19. Neish AS: Microbes in gastrointestinal health and disease. Gastroenterology 136: 65-80, 2009.

20. Hussain QA, McKay IJ, Gonzales-Marin C and Allaker RP: Regulation of adrenomedullin and nitric oxide production by periodontal bacteria. J Periodontal Res 50: 650-657, 2015.

21. Sadeghi-Aliabadi H, Mohammadi F, Fazeli H and Mirlohi M: Effects of Lactobacillus plantarum A7 with probiotic potential on colon cancer and normal cells proliferation in comparison with a commercial strain. Iran J Basic Med Sci 17: 815-819, 2014.
22. Liu L, Liang L, Liang H, Wang M, Lu B, Xue M, Deng J and Chen Y: Fusobacterium nucleatum aggravates the progression of colitis by regulating M1 macrophage polarization via AKT2 pathway. Front Immunol 10: 1324, 2019.

23. Liu H, Hong X, Sun T, Huang X, Wang J and Xiong H: Fusobacterium nucleatum exacerbates colitis by damaging epithelial barrier and inducing aberrant inflammation. J Dig Dis: May 22, 2020 (Epub ahead of print). doi: 10.1111/1751-2980.12909.

24. Cooper HS, Murthy SN, Shah RS and Sedergran DJ: Clinicopathologic study of dextran sulfate sodium experimental murine colitis. Lab Invest 69: 238-249, 1993.

25. Dutton JW III, Artwohl JE, Huang $X$ and Fortman JD: Assessment of pain associated with the injection of sodium pentobarbital in laboratory mice (Mus musculus). J Am Assoc Lab Anim Sci 58: 373-379, 2019.

26. Livak KJ and Schmittgen TD: Analysis of relative gene expression data using real-time quantitative PCR and the 2(-Delta Delta C(T)) method. Methods 25: 402-408, 2001.

27. Zhang Q, Gao M, Zhang Y, Song Y, Cheng H and Zhou R: The germline-enriched Ppp1r36 promotes autophagy. Sci Rep 6: 24609, 2016.

28. Cardiff RD, Miller CH and Munn RJ: Manual hematoxylin and eosin staining of mouse tissue sections. Cold Spring Harbor protocols 2014: 655-658, 2014.

29. Horino J, Fujimoto M, Terabe F, Serada S, Takahashi T, Soma Y, Tanaka K, Chinen T, Yoshimura A, Nomura S, et al: Suppressor of cytokine signaling-1 ameliorates dextran sulfate sodium-induced colitis in mice. Int Immunol 20: 753-762, 2008.

30. Misra RM, Bajaj MS and Kale VP: Vasculogenic mimicry of HT1080 tumour cells in vivo: Critical role of HIF-1 $\alpha$-neuropilin-1 axis. PLoS One 7: e50153, 2012.

31. Baxt LA and Xavier RJ: Role of Autophagy in the Maintenance of Intestinal Homeostasis. Gastroenterology 149: 553-562, 2015.

32. Heras-Sandoval D, Pérez-Rojas JM, Hernández-Damián J and Pedraza-Chaverri J: The role of PI3K/AKT/mTOR pathway in the modulation of autophagy and the clearance of protein aggregates in neurodegeneration. Cell Signal 26: 2694-2701, 2014.

33. Kostic AD, Chun E, Robertson L, Glickman JN, Gallini CA, Michaud M, Clancy TE, Chung DC, Lochhead P, Hold GL, et al: Fusobacterium nucleatum potentiates intestinal tumorigenesis and modulates the tumor-immune microenvironment. Cell Host Microbe 14: 207-215, 2013.

34. Shen ZH, Zhu CX, Quan YS, Yang ZY, Wu S, Luo WW, Tan B and Wang XY: Relationship between intestinal microbiota and ulcerative colitis: Mechanisms and clinical application of probiotics and fecal microbiota transplantation. World J Gastroenterol 24: 5-14, 2018.

35. Boonma P, Spinler JK, Venable SF, Versalovic J and Tumwasorn S: Lactobacillus rhamnosus L34 and Lactobacillus casei L39 suppress Clostridium difficile-induced IL-8 production by colonic epithelial cells. BMC Microbiol 14: 177, 2014

36. Johnson-Henry KC, Donato KA, Shen-Tu G, Gordanpour M and Sherman PM: Lactobacillus rhamnosus strain GG prevents enterohemorrhagic Escherichia coli O157:H7-induced changes in epithelial barrier function. Infect Immun 76: 1340-1348, 2008.

37. Macias-Ceja DC, Cosin-Roger J, Ortiz-Masiá D, Salvador P, Hernández C, Esplugues JV, Calatayud S and Barrachina MD: Stimulation of autophagy prevents intestinal mucosal inflammation and ameliorates murine colitis. Br J Pharmacol 174: 2501-2511, 2017.

38. Nguyen HT, Dalmasso G, Müller S, Carrière J, Seibold F and Darfeuille-Michaud A: Crohn's disease-associated adherent invasive Escherichia coli modulate levels of microRNAs in intestinal epithelial cells to reduce autophagy. Gastroenterology 146: 508-519, 2014.

This work is licensed under a Creative Commons Attribution-NonCommercial-NoDerivatives 4.0 International (CC BY-NC-ND 4.0) License. 